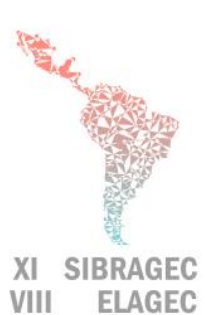

VIII ELAGEC
XI SIMPÓSIO BRASILEIRO DE GESTÃO E ECONOMIA DA CONSTRUÇÃO

VIII ENCUENTRO LATINOAMERICANO DE GESTIÓN Y ECONOMÍA DE LA CONSTRUCCIÓN

Del conocimiento a la acción: prácticas avanzadas de gestión de la producción

Londrina, Paraná, Brasil. 23 al 25 de Octubre del 2019

\title{
ESTRATEGIAS DE INTERNACIONALIZACIÓN DE LAS EMPRESAS CONSULTORAS DE INGENIERÍA ESPAÑOLAS
}

\author{
LOZANO-TORRÓ, Alicia (1); PELLICER, Eugenio (2); SANZ, Amalia (3); \\ JIMÉNEZ, Jaime (4)
}

(1) Universidad Politécnica de Valencia (España),+34963879562, email: allotor@ upv.es (2) Universidad Politécnica de Valencia (España), email: pellicer@upv.es (3) Universidad Politécnica de Valencia (España), email: asanz@ upv.es (4) Universidad Politécnica de Valencia (España), email: jaijiay@upv.es

\begin{abstract}
El contexto de crisis económica en España en el periodo 2008-2013 y el entorno globalizado actual han convertido la internacionalización en una de las principales vías de supervivencia para las ingenierías españolas. Pero los elevados riesgos que implica el mercado internacional obligan a las ingenierías a la adopción de determinadas decisiones estratégicas. El objetivo del presente artículo es analizar las estrategias de internacionalización que las ingenierías españolas consideran más importantes para obtener establecerse con éxito en mercado externos. Partiendo de las estrategias identificadas en la revisión de la literatura se entrevistan 10 directivos de ingenierías españolas con experiencia internacional. Los resultados muestran que las estrategias más valoradas son la relacionada con la estructura, que debe modificarse para hacerla más flexible, en la que prevalecen ventajas tales como la disposición de recursos humanos cualificados, elaboración de trabajos de alta calidad, prestación de servicios especializados y la adquisición de experiencia en el mercado nacional. Además, el tamaño de las empresas favorece la internacionalización porque facilita la obtención de ciertas ventajas competitivas, como unos recursos humanos altamente cualificados o una mayor capacidad financiera. Estas conclusiones facilitan el proceso de internacionalización de las empresas de ingeniería, sobre todo en un contexto de crisis económica.
\end{abstract}

Keywords: Estrategias de internacionalización, ventajas competitivas, mercado internacional, ingenierías españolas, éxito

\section{INTRODUCCIÓN}

\subsection{Planteamiento del problema}

La mejora de las economías de los países en desarrollo (ZHAO et al., 2017) y el contexto globalizado actual han favorecido la internacionalización de las empresas del sector de la construcción (YE; LU; JIANG, 2009). Sin embargo, el mercado internacional conlleva más riesgos que el nacional, debido a las diferencias políticas, económicas, sociales y culturales respecto al país de origen (HAN et al., 2007; JAVERNICK-WILL; SCOTT, 2010; JIN et al., 2013; PARK et al., 2014). Para hacer 
frente a estos riesgos los contratistas internacionales tienen que adoptar decisiones estratégicas que les permitan posicionarse en el mercado global (JIN et al., 2013) y disponer de ciertas ventajas competitivas para una mayor adaptación a estos entornos desconocidos (MOSLEY; BUBSHAIT, 2016). Para Larrinaga (2008), gran parte del éxito de la internacionalización de una empresa es producto de una adecuada dirección estratégica. Asimismo el desempeño estratégico, que mide las estrategias y determina la influencia de los resultados en los objetivos de la empresa, es un importante indicador para determinar si las empresas obtendrán una ventaja competitiva frente a sus competidores (ERCAN; KOKSAL, 2016).

\subsection{Contexto de la investigación}

A pesar de la incertidumbre que genera el mercado internacional, existen varios motivos por los que los contratistas deciden acometer su actividad en el exterior: saturación del mercado nacional, distribución del riesgo o aprovechamiento de las oportunidades en el exterior (ALASHWAL et al., 2017). Es la saturación del mercado nacional, producto de la crisis económica española iniciada en el año 2007, uno de los principales motivos que llevó a las ingenierías españolas a internacionalizarse (TECNIBERIA, 2011). Esta situación se vio agravada por el elevado volumen de negocios y expansión de las ingenierías en los años previos a la crisis, originando estructuras de operativas rígidas con dificultad para adaptarse a reducciones del mercado (TECNIBERIA, 2011). Debido a la importancia de entender las variables que conducen al éxito internacional (ALASHWAL et al., 2017) se han realizado varios estudios que analizan la internacionalización de las empresas de ingeniería españolas: García-Canal et al. (2002) investigaron las alianzas globales como estrategia para acelerar el ritmo de la internacionalización; García et al. (2007) analizaron el modo de entrada como parte de la estrategia de internacionalización de las empresas españolas de ingeniería, y Goñi (2008) identificó las razones, modos de entrada, ventajas competitivas y barreras en la internacionalización de las ingenierías españolas en el periodo 1993-2008. Sin embargo, no se han desarrollado investigaciones que identifiquen las principales estrategias de internacionalización de las ingenierías españolas, en un contexto de crisis económica, para conseguir éxito en su actividad exterior.

\subsection{Objetivos}

El presente artículo tiene como objetivo analizar las estrategias más importantes para las empresas de ingeniería españolas en su actividad internacional, partiendo de las estrategias analizadas en la literatura en el periodo 1976-2017.

\section{MÉTODO DE INVESTIGACIÓN}

La primera parte del estudio identifica las estrategias de internacionalización de las empresas constructoras mediante la revisión de artículos comprendidos en el periodo 1976-2017. Se han identificado varias investigaciones que analizan las estrategias de internacionalización de las empresas del sector de la construcción: Krull et al. (2012) analizaron la estrategia de una ingeniería neozelandesa con 40 años de experiencia internacional desde la perspectiva de la industria, de los recursos y capacidades de la empresa y de los factores institucionales. Zhao et al. (2009) estudiaron las fortalezas, debilidades oportunidades y amenazas de los contratistas internacionales chinos con el fin de seleccionar estrategias que les permitiesen reforzar sus posiciones en el mercado internacional. Tiong y Yeo (1993) identificaron los obstáculos a los que tienen 
enfrentarse los contratistas para financiar sus proyectos internacionales, proponiendo como solución estrategias competitivas y de entrada. Xu y Greenwood (2006) investigaron las estrategias de entrada de los contratistas extranjeros en China en contratos de diseño y construcción.

Partiendo del modelo estratégico propuesto por Larrinaga (2008), en el que la formulación de la estrategia de internacionalización se divide en 10 ámbitos estratégicos, Lasús (2011) adaptó este modelo al caso de las consultoras de ingeniería españolas, donde reduce los ámbitos estratégicos a siete: localización, entrada, corporativa, internalización, competitiva, estructura y secuencia (Figura 1).

\section{Figura 1 - Modelo estratégico de internacionalización adaptado a las}

\section{ingenierías españolas}

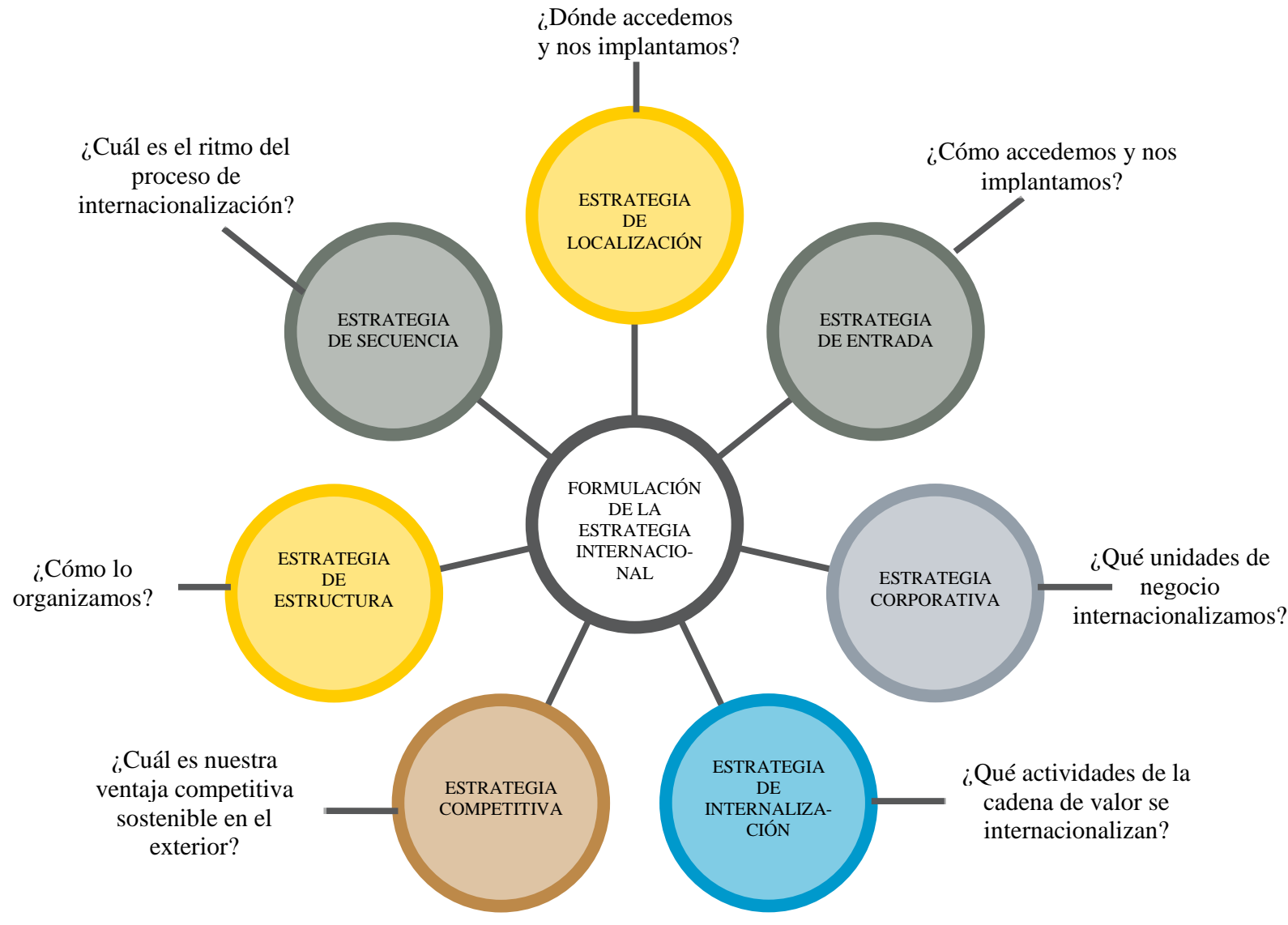

Fuente: Lasús (2011)

La estrategia de localización está estrechamente ligada al país de destino (LARRINAGA, 2008), analizándose variables como el idioma, la estabilidad política y social, la seguridad jurídica, los clientes, la inversión en el sector o la competencia (ARRÓSPIDE; MACÍAS, 2012). En la estrategia de entrada intervienen aspectos relacionados con los recursos y capacidades de la empresa, pero también con los países de origen y destino (LASÚS, 2011). Por su parte, en el ámbito de la estrategia corporativa se tiene en cuenta el posicionamiento en el mercado, la cuota y tasa de crecimiento y el nivel de madurez del producto que vamos a internacionalizar 
(ARRÓSPIDE; MACÍAS, 2012). Relacionada con esta estrategia se encuentra la diversificación, entendida como "una forma de estrategia que se aplica para aumentar la rentabilidad mediante el movimiento rápido de una empresa en muchos mercados y productos extranjeros" (DANIELS; RADEBAUGH; SULLIVAN, 1998), pudiendo ser regional o de producto. En la estrategia de internalización se decide si en el exterior se realizan todas o algunas de las actividades primarias de la cadena de valor de la empresa (LARRINAGA, 2008), decidiendo si la empresa adoptará una expansión horizontal o vertical. La estrategia competitiva tiene en cuenta la integración global, cuyo objetivo es reducir costes y coordinar de forma centralizada las actividades y los precios de los productos en distintos países, o la adaptación local, que se adapta a las peculiaridades de cada país proporcionando una elevada capacidad de respuesta local y una minimización de los riesgos político y de cambio (LÓPEZ NAVARRETE, 2013). En la estrategia de estructura, la empresa analiza la estructura organizativa más adecuada para desarrollar la actividad internacional (LARRINAGA, 2008), abandonando las estructuras tradicionales (STEFFEY; ANANTATMULA, 2011) y adaptándose a las nuevas necesidades conforme aumenta el grado de internacionalización (LASÚS, 2011). Finalmente, en la estrategia de secuencia, la empresa analizará si adopta una estrategia desarrollando un proceso gradual a medio/largo plazo o si elige una estrategia simultánea optando por una internacionalización acelerada, como es el caso de las empresas "born" global (LARRINAGA, 2008).

La segunda parte de la investigación tiene como objetivo analizar las estrategias de internacionalización que las ingenierías españolas consideran más importantes, teniendo en cuenta el contexto de crisis económica español. Con el objeto de validar las variables identificadas en la literatura se diseñó una entrevista semiestructurada (WOODSIDE, 2010) como herramienta de investigación cualitativa, y se envió a 10 ejecutivos de empresas de ingeniería españolas, elegidas por accesibilidad mediante un muestreo no probabilístico de conveniencia adecuado para estudios exploratorios (CARRIÓN, 2013). La selección de las empresas trató de cubrir diferentes características, tal y como muestra la Tabla 1.

Tabla 1 - Características de las empresas entrevistadas

\begin{tabular}{|c|c|c|c|c|c|c|c|c|c|c|c|}
\hline \multirow{2}{*}{\multicolumn{2}{|c|}{ Característica }} & \multicolumn{10}{|c|}{ Empresa } \\
\hline & & \multirow{2}{*}{ E1 } & \multirow[t]{2}{*}{ E2 } & \multirow[t]{2}{*}{ E3 } & \multirow{2}{*}{\begin{tabular}{|c|}
$\mathbf{E 4}$ \\
$X$
\end{tabular}} & \multirow[t]{2}{*}{ E5 } & \multirow{2}{*}{ E6 } & \multirow{2}{*}{ E7 } & \multirow{2}{*}{$\frac{\mathbf{E 8}}{X}$} & \multirow{2}{*}{ E9 } & \multirow[t]{2}{*}{ E10 } \\
\hline Tamaño & Multinacional & & & & & & & & & & \\
\hline & PYME & & & $\mathrm{X}$ & & $\mathrm{X}$ & $\mathrm{X}$ & $\mathrm{X}$ & & $\mathrm{X}$ & $\mathrm{X}$ \\
\hline & Microempresa & $\mathrm{X}$ & $\mathrm{X}$ & & & & & & & & \\
\hline \multicolumn{2}{|c|}{$\begin{array}{l}\text { Año de inicio de la } \\
\text { internacionalización }\end{array}$} & 2012 & 2013 & 2011 & 1975 & 2007 & 2006 & 2003 & 2005 & 2011 & 2008 \\
\hline \multirow{5}{*}{$\begin{array}{l}\text { Área de } \\
\text { actividad }\end{array}$} & Ingeniería civil & & $\mathrm{X}$ & $\mathrm{X}$ & $\mathrm{X}$ & $\mathrm{X}$ & $\mathrm{X}$ & $\mathrm{X}$ & $\mathrm{X}$ & $\mathrm{X}$ & $\mathrm{X}$ \\
\hline & Edificación & & & $\mathrm{X}$ & $\mathrm{X}$ & $\mathrm{X}$ & $\mathrm{X}$ & $\mathrm{X}$ & $\mathrm{X}$ & $\mathrm{X}$ & $\mathrm{X}$ \\
\hline & Medio ambiente & & $\mathrm{X}$ & $\mathrm{X}$ & $\mathrm{X}$ & $\mathrm{X}$ & $\bar{X}$ & & $\mathrm{X}$ & $\mathrm{X}$ & \\
\hline & Industria y energía & $\mathrm{X}$ & & $\mathrm{X}$ & $\mathrm{X}$ & & & & $\mathrm{X}$ & $\mathrm{X}$ & \\
\hline & Servicios & $\mathrm{X}$ & & & & & $\mathrm{X}$ & & $\mathrm{X}$ & & \\
\hline
\end{tabular}

Fuente: Elaboración propia 
La entrevista comprendió dos partes: (1) caracterización de los entrevistados, y (2) el cuestionario. Para determinar la importancia de las estrategias de internacionalización se utilizó una escala tipo Likert de 11 puntos (donde $0=$ "totalmente en desacuerdo" y $10=$ "totalmente de acuerdo"). Las 10 entrevistas se transcribieron a una hoja de cálculo y se utilizó el programa estadístico SPSS versión 24 para analizar los datos obtenidos.

\section{RESULTADOS}

Los resultados de las entrevistas obtenidos en la pregunta relacionada con el grado de acuerdo o desacuerdo de las empresas con las estrategias de internacionalización, partiendo de los ámbitos estratégicos propuestos por Lasús (2011) se muestran en la Tabla 2.

Tabla 2 - Estrategias de internacionalización. Estadísticos descriptivos

\begin{tabular}{|l|c|c|c|}
\hline \multicolumn{1}{|c|}{ Estrategia } & Media & Mediana & Desviación \\
\hline Localización & 6.8 & 6.5 & 3,05 \\
\hline Entrada & 7.0 & 7.5 & 2,94 \\
\hline Corporativa & 5.5 & 5.0 & 3,03 \\
\hline Internalización & 6.7 & 8.0 & 2,87 \\
\hline Competitiva & 7.6 & 8.5 & 3,17 \\
\hline Estructura & 7.8 & 8.5 & 2,90 \\
\hline Secuencia & 5.9 & 6.0 & 2,73 \\
\hline
\end{tabular}

Fuente: Elaboración propia

Todas las estrategias tienen medias iguales o superiores a 5.5, siendo las más valoradas las estrategias de estructura y la competitiva, seguidas de las de entrada y de localización. Respecto a la estrategia de estructura, este resultado implica que las empresas consideran muy importante disponer de una estructura organizativa adecuada para acometer la actividad internacional, incrementando su flexibilidad (E1), creando un departamento de licitaciones internacionales o contratando técnicos con movilidad al exterior (E3), ampliando los sistemas de gestión de proyectos, administración, asesoría laboral, contable y fiscal para adaptarse a las nuevas necesidades de la actividad internacional (E6 y E10) o perfeccionando los perfiles internacionales (E8). Por su parte, en la estrategia competitiva se definen las ventajas competitivas sostenibles de la empresa en el exterior, siendo las más valoradas por las empresas el disponer de recursos humanos cualificados, prestar trabajos de alta calidad, prestar servicios especializados y personalizados y la experiencia en el mercado nacional, seguidas de una buena relación y atención al cliente. Para las empresas E3 y E4 es importante disponer de recursos humanos cualificados del país de origen con movilidad al exterior, ya que los técnicos españoles tienen un perfil generalista que facilita la flexibilidad y adaptación a los cambios de los proyectos (E4). Respecto a la influencia del tamaño de la empresa, el $90 \%$ de las empresas entrevistadas afirman que aumenta la posibilidad de obtener ventajas competitivas como la capacidad financiera (E4 y E10), el obtener recursos humanos cualificados (E3 y E6) o una mejor reputación (E7). Sin embargo, la empresa E5 considera que el tamaño de la empresa influye de forma negativa en la atención al cliente o en la prestación de servicios personalizados. Finalmente, las estrategias menos valoradas han sido la estrategia corporativa y la de secuencia, es 
decir, que la decisión de internacionalizar algunas o todas las unidades de negocio y adoptar una internacionalización progresiva o simultánea no influirá de forma determinante en el éxito de internacionalización de las ingenierías españolas.

\section{CONCLUSIONES}

A través de la revisión de la literatura en el periodo 1976-2017 se han identificado las principales estrategias de internacionalización llevadas a cabo por las empresas del sector de la construcción, validadas mediante la realización de 10 entrevistas a ingenierías españolas con experiencia internacional, las cuales se internacionalizaron para asegurar su supervivencia ante la crisis económica iniciada en España en el año 2007. Los resultados muestran que las estrategias más valoradas son: la relacionada con la estructura y la estrategia competitiva, lo que implica que las empresas consideran que deben reorganizarse estructuralmente para aumentar su flexibilidad en el exterior. Las principales ventajas competitivas que deben adquirir las empresas para obtener éxito son: la disposición de recursos humanos cualificados, prestar trabajos de alta calidad, prestar servicios especializados y personalizados y la experiencia en el mercado nacional. Se constata que el tamaño de una empresa favorece la obtención de ventajas competitivas, como la capacidad financiera y la disposición de recursos humanos cualificados. Finalmente la estrategia corporativa y la de secuencia se consideran las menos importantes por las empresas para conseguir éxito internacional.

\section{REFERENCIAS}

ALASHWAL, Ali Mohammed; FAREED, Nurfarhana Farza; AL-OBAIDI, Karam Mustafa. Determining success criteria and success factors for international construction projects for Malaysian contractors. Construction Economics and Building, v. 17, n. 2, p. 62-80, June 2017.

ARRÓSPIDE, Gonzalo López-Oleaga; MACÍAS, Carlos Raúl Collado. Planificación y gestión operativa internacional de una empresa de ingeniería industrial. 2012. Proyecto Fin de Carrera, Escuela Técnica Superior de Ingeniería (ICAI), Universidad Pontificia de Comillas, Madrid.

CARRIÓN, A. Técnicas Estadísticas de Investigación Social. Universitat Politècnica de València, Valencia, 2013.

DANIELS, John D.; RADEBAUGH, Lee H.; SULLIVAN, Daniel P. International Business: Environments and Operations. Addison-Wesley, 1998.

ERCAN, Tugce; KOKSAL, Almula. Competitive Strategic Performance Benchmarking (CSPB) model for international construction companies. KSCE Journal of Civil Engineering, v. 20, n. 5, p. 1657-1668, July 2016.

GARCÍA-CANAL, Esteban, et al. Accelerating international expansion through global alliances: A typology of cooperative strategies. Journal of World Business, v. 37, n. 2, p. 91107, 2002.

GARCÍA, Haydée Calderón, et al. Selección del modo de entrada en un mercado internacional: valoración de las capacidades empresariales, la estrategia empresarial y la percepción de los problemas de la internacionalización. ICE, Revista de Economía, n. 839, dic. 2007. Disponible en: http://www.revistasice.org/index.php/ICE/article/view/1104. Acceso en: 23 febrero 2019, 18:10:45.

GOÑI, Manuel García. La Internacionalización de los servicios de ingeniería en España. ICE, Revista de Economía, n. 844, oct. 2008. Disponible en: 


\section{SIBRAGEC - ELAGEC 2019 - del 23 al 25 de Octubre - LONDRINA - PR}

http://www.revistasice.org/index.php/ICE/article/view/1104. Acceso en: 23 febrero 2019, 18:40:15.

HAN, Seung H., et al. Causes of bad profit in overseas construction projects. Journal of Construction Engineering and Management, v. 133, n. 12, p. 932-943, Dec. 2007.

JAVERNICK-WILL, Amy N.; SCOTT, W. Richard. Who needs to know what? Institutional knowledge and global projects. Journal of Construction Engineering and Management, v. 136, n. 5, p. 546-557, Apr. 2010.

JIN, Zhigang, et al. Practical framework for measuring performance of international construction firms. Journal of Construction Engineering and Management, v. 139, n. 9, p. 1154-1167, Mar. 2013.

KRULL, Elisabeth; SMITH, Peter; GE, Gloria L. The internationalization of engineering consulting from a strategy tripod perspective. The Service Industries Journal, v. 32, n. 7, p. 1097-1119, Mar. 2012.

LARRINAGA, Oskar Villarreal, et al. La internacionalización de la empresa: el modelo de las diez estrategias. Revista Internacional Administración y Finanzas, v. 1, n.1, p. 67, 2008.

LASÚS, J. F. Plan de Internacionalización de una ingeniería: modelo teórico y caso práctico. 2011. Doctoral dissertation, Departament d'Infraestructura del Transport i del Territori, Escola Tècnica Superior d'Enginyers de Camins, Canals i Ports de Barcelona, Universitat Politècnica de Catalunya, Barcelona, 2011.

LÓPEZ NAVARRETE, ALEJANDRO. Internacionalización de la pequeña y mediana empresa de ingeniería española, una alternativa de futuro. Presentación de un caso de éxito: SEG Colombia. Trabajo Final de Máster. Universitat Politècnica de València. Facultad de Administración y Dirección de Empresas - Facultat d'Administració i Direcció d'Empreses, Valencia, 2013.

MOSLEY, John C.; BUBSHAIT, Abdulaziz A. Performance evaluation of international contractors in Saudi Arabia. Journal of Professional Issues in Engineering Education and Practice, v. 142, n. 1, p. 04015007, Apr. 2015.

PARK, Heedae, et al. Effect of institutional risks on the performance of international construction projects. In: CONSTRUCTION RESEARCH CONGRESS, 2014, Atlanta, Georgia: Construction in a Global Network, 2014. p. 2126-2135.

STEFFEY, Robert W.; ANANTATMULA, Vittal S. International projects proposal analysis: Risk assessment using radial maps. Project Management Journal, v. 42, n. 3, p. 62-74, Apr. 2011.

TECNIBERIA. Desarrollo Estratégico del Sector de la Ingeniería de Consulta. 2011. Disponible en: http://www. tecniberia.es/documentos/Desarrollo.pdf. Acceso en: 23 febrero 2019, 17:30:30.

TIONG, R.; YEO, K. T. Project financing as a competitive strategy in winning overseas jobs. International Journal of Project Management, v. 11, n. 2, p. 79-86, May 1993.

WOODSIDE, Arch G. Case Study Research: Theory, Methods and Practice. Emerald Group Publishing, 2010.

XU, Tianji; GREENWOOD, David. Using design-and-build as an entry strategy to the Chinese construction market. International Journal of Project Management, v. 24, n. 5, p. 438-445, July 2006.

YE, Kunhui; LU, Weisheng; JIANG, Weiyan. Concentration in the international construction market. Construction Management and Economics, v. 27, n. 12, p. 1197-1207, Dec. 2009. 
SIBRAGEC - ELAGEC 2019 - del 23 al 25 de Octubre - LONDRINA - PR

ZHAO, Zhen Yu; SHEN, Li Yin; ZUO, Jian. Performance and strategy of Chinese contractors in the international market. Journal of Construction Engineering and Management, v. 135, n. 2, p. 108-118, Feb. 2009.

ZHAO, Zhen-Yu, et al. Agglomeration and competitive position of contractors in the international construction sector. Journal of Construction Engineering and Management, v. 143, n 6, p. 04017004, Feb. 2017. 